\title{
SEGMENTATION OF ELECTROENCEPHALOGRAM SIGNALS DURING EPILEPTIC SEIZURES BY USING FUZZY C-MEANS
}

\author{
${ }^{1,2}$ Muhammad Abdy and ${ }^{3}$ Tahir Ahmad \\ ${ }^{1}$ Department of Mathematics, Universitas Negeri Makassar, Indonesia \\ ${ }^{2}$ Fakultas MIPA, Universitas Sulawesi Barat, Indonesia \\ ${ }^{3}$ Department of Mathematical Sciences and Ibnu Sina Institute for Fundamental Science Studies, \\ Universiti Teknologi Malaysia, 81310 Skudai, Johor Darul Takzim, Malaysia
}

Received 2014-07-16; Revised 2014-10-02; Accepted 2014-11-06

Funding: The Government of South Sulawesi Province, Indonesia and Universiti Teknologi Malaysia

Competing Interests: The authors have declared that no competing interests exist

\begin{abstract}
Electroencephalogram (EEG) is a recording of electrical activity of the brain. It contains valuable information related to the different physiological states of the brain. A quantitative EEG analysis has been developed over the years that introduce objective measure, reflecting not only the characteristics of the brain activity itself but also giving clues concerning the underlying associated neural dynamics. In this study, the image form of the EEG signals is segmented into parts that have the nearly equal electrical current strength. This segmentation uses fuzzy c-means. An example of EEG signal data will be provided and segmented using the obtained method.
\end{abstract}

Keywords: EEG Signals, Fuzzy-C-Mean, Segmentation

\section{INTRODUCTION}

Epileptic seizures result from a temporary electrical disturbance of the brain. Sometimes seizures may go unnoticed, depending on their presentation and sometimes may be confused with other events, such as a stroke, which can also cause falls or migraines (Subasi, 2005). Epileptic seizures may cause negative physical, psychological and social consequences, including loss of consciousness, injuries and sudden death (Guo et al., 2010). Prediction of seizures is challenging because there is very little confirmed knowledge of the exact mechanism responsible for the seizure. Effective algorithms for automatic seizure detection and prediction can have a far reaching impact on diagnosis and treatment of epilepsy (Adeli, 2007). Surgical treatment may be an option in patients having epileptic seizures refractory to medication (Engel et al., 1993). The main problem in epilepsy surgery is to resolve the size and location of the epileptic foci. Therefore, it is crucial to determine an accurate technique which is capable to localize the epileptic focus in patient who is suffering from epilepsy.

The Electroencephalogram (EEG) is a resultant signal of the active potentials of many nerve cells in the cerebral cortex and captures the cerebral function. EEG is one of the irregular and feeble signals in the living body (Zhanga et al., 2001). It plays an important diagnostic role in epilepsy and provides supporting evidence of a seizure disorder as well as assisting with classification of seizures. Careful analysis of the EEG records can provide new insights into the epileptogenic process and may have considerable utilization in the diagnosis and treatment of epilepsy (Binjadhnan and Ahmad, 2010).

Corresponding Authors: Tahir Ahmad, Ibnu Sina Institute for Fundamental Science Studies, Universiti Teknologi Malaysia, 81310 Skudai, Johor, Malaysia and Muhammad Abdy, Department of Mathematics, Universitas Negeri Makassar, Indonesia 
In recent years, some researchers have studied on seizure detection and prediction from EEG analysis using two different approaches: (1) Examination of the waveforms of the EEG signals such as spikes, which may be precursors to seizures; (2) Analysis of the nonlinear spatiotemporal evolution of the EEG signals to find a governing rule as the system moves from a seizure-free to seizure state (Adeli, 2007). All of the methods used to analyze the neuronal activities in the brain during epileptic seizures using EEG signals in the wave form. In the work of Abdy (2014), the neuronal activities in the brain during epileptic seizures displayed on flat EEG were transformed into image form. The flat EEG is a new method to map high dimensional EEG signal into low dimensional space (Zakaria, 2008). Seizure and the flat EEG that are modeled as a dynamical systems share the same dynamics. Besides, their augmented dynamic trajectory is linearly ordered and order isomorphic to each other by the relation induced from their motion (Ahmad and Ken, 2010). In this study, image form of the EEG signals during epileptic seizures is segmented into regions using fuzzy c-mean.

\section{MATERIALS AND METHODS}

\subsection{Image form of EEG Signals}

Let $C$ be all the cluster centers at time $t$, i.e.: $C_{t}=\left\{c_{1}\right.$, $\left.c_{2}, \ldots, c_{m}\right\} ; m=$ the number of cluster center at time $t$. Each $c_{j}$ carries the position a flat EEG and electrical potential, in short:

$$
\left(\mathrm{c}_{\mathrm{j}}\right)_{\mathrm{t}}=\left((x, y), V_{j}\right)_{t}
$$

$V_{j}=$ The electrical potential of the cluster center $j^{\text {th }}$, so

$C_{t}=\left\{\left((x, y), V_{j}\right)_{t} \mid x, y, V_{j} \in \mathbb{R}\right\}$

where, $j=1,2, \ldots, m$.

Let $P$ be the entire pixels of the flat EEG, i.e.:

$$
P=\left\{p_{1}, p_{2}, \ldots p_{n}\right\} \text { such that } p_{i}=(x, y), \text { then } C_{t} \subset P
$$

A fuzzy neighborhood can be defined as a degree $\mu_{x}(y)$ to which two points $x$ and $y$ are neighbors. Several definitions have been proposed (Demko and Zahzah, 1995; Bloch et al., 1997), which are typically decreasing functions of the distance between both points. For purpose of forming image at flat EEG, we consider a fuzzy neighborhood of electrical potential for cluster center $c_{j}$ at each pixel $p_{i}$ at time $t$. The fuzzy neighborhood is represented by a membership function $\mu_{c j}$. The memberships function $\mu_{c_{j}}$ at time $t$ proposed by Abdy (2014) as Equation 1 and 2:

$\mu_{c_{j}}\left(p_{i}\right)_{t}=\frac{\left(V_{j}\right)_{t}}{\left(V_{j}\right)_{t}+d\left(p_{i}, c_{j}\right)_{t}}$

where, $\left(V_{j}\right)_{t}$ is the electrical potential $c_{j}$ at time $t$.

And:

$d\left(p_{i}, c_{j}\right)_{t}=\sqrt{\left(\left(x_{c_{j}}-x_{i}\right)^{2}+\left(y_{c_{j}}-y_{i}\right)^{2}\right)_{t}}$

is distance between pixel $p_{i}$ and cluster center $\mathrm{c}_{\mathrm{j}}$ at time $t$. Standardization will be done to $V_{j}$ and $d\left(p_{i}, p_{j}\right)$ so they will free from unit (dimensionless quantity). Standardization of $V_{j}$ and $d\left(p_{i}, p_{j}\right)$ as follows (Abdy, 2014) Equation 3:

$\left(V_{j}\right)^{\prime}=\frac{V_{j}-\bar{V}_{j}}{\sigma_{V_{j}}}$ and $\left(d\left(p_{i}, c_{j}\right)\right)^{\prime}=\frac{d\left(p_{i}, c_{j}\right)-\overline{d\left(p_{i}, c_{j}\right)}}{\sigma_{d\left(p_{i}, c_{j}\right)}}$

Due to $\left(V_{j}\right)^{\prime}$ and $\left(d\left(p_{i}, c_{j}\right)^{\prime}\right.$ must be positive and greater or equal to 0 , respectively, we transform them into $(0, a]$ and $[0, b]$, respectively. This transformation can be accomplished by applying a linear mapping function as given below (Abdy, 2014) Equation 4 to 6:

$\left(V_{j}\right)^{\prime \prime}=\frac{\left|\left(V_{j}\right)_{\min }^{\prime}\right|+\left|\left(V_{j}\right)_{\max }^{\prime}\right|}{\left(V_{j}\right)_{\max }^{\prime}-\left(V_{j}\right)_{\min }^{\prime}}\left(\left(V_{j}\right)^{\prime}-\left(V_{j}\right)_{\min }^{\prime}\right)+\varepsilon ; \varepsilon>0$

And:

$$
\begin{aligned}
\left(d\left(p_{i}, c_{j}\right)\right)^{\prime \prime} & =\frac{\left|\left(d\left(p_{i}, c_{j}\right)\right)_{\min }^{\prime}\right|+\left|\left(d\left(p_{i}, c_{j}\right)\right)_{\max }^{\prime}\right|}{\left(d\left(p_{i}, c_{j}\right)\right)_{\max }^{\prime}-\left(d\left(p_{i}, c_{j}\right)\right)_{\min }^{\prime}} \\
& \times\left(\left(d\left(p_{i}, c_{j}\right)\right)^{\prime}-\left(d\left(p_{i}, c_{j}\right)\right)_{\min }^{\prime}\right)^{\prime}
\end{aligned}
$$

Hence:

$$
\mu_{c_{j}}\left(p_{i}\right)=\frac{\left(V_{j}\right)^{\prime \prime}}{\left(V_{j}\right)^{\prime \prime}+\left(d\left(p_{i}, c_{j}\right)\right)^{\prime \prime}}
$$

If it is considered that the fuzzy neighborhood for each cluster center $c_{j}$ at time $t$ form a fuzzy region of 
the electric current at flat EEG, then $P$ is a fuzzy set whose membership value $\mu_{P}\left(p_{i}\right)_{t}$ of each its elements, i.e., Equation 7:

$$
P_{t}=\left\{\left((x, y), \mu_{P}\left(p_{i}\right)_{t}\right) \mid x, y \in \mathbb{R}, \mu_{P}\left(p_{i}\right)_{t} \in[0,1]\right\}
$$

The fuzzy set $P_{t}$ constitutes union of the fuzzy neighborhood of the cluster centers at time $t$. Hence, it can be obtained the membership value $\mu_{P}\left(p_{i}\right)_{t}$ of each pixel $p_{i}$ by using the operation of union (max operator), i.e., Equation 8:

$$
\mu_{P}\left(p_{i}\right)_{t}=\max _{j}\left[\mu_{c_{j}}\left(p_{i}\right)_{t}\right]
$$

Each pixel within a fuzzy region of flat EEG can be interpreted as degree to which that pixel is a current source at flat EEG. Next, the membership value of each pixel $p_{i}$ is transformed into the image data $I$. This is done so we can see the sources of electricity in flat EEG as grey levels. Each pixel will be represented by an integer value in the interval 0 to 255 . The membership value 0 is mapped to 0 in the image data and the membership value 1 mapped into the image data 255. The transformation that is used at each pixel $p_{i}$ into the image data $I$ is a linear function (Abdy, 2014), namely Equation 9:

$$
I_{t}=255 \mu_{P}\left(p_{i}\right)_{t}
$$

Hence, it is obtained the image form of EEG signals at the flat EEG during epileptic seizure.

\subsection{Segmentation}

Segmentation, one of the bottlenecks of medical image processing, is a fundamental building block for higher-level image analysis; it allows a compact description of the image into contours, regions. The segmentation actually attempts to discover associations between subclasses of a population to reduce the dimension (Demko and Zahzah, 1995). There are two main approaches used in segmentation which are based on crisp and fuzzy methods. Crisp segmentation algorithm generates partitions such that each object is assigned to exactly one class; on the other hand, fuzzy methods provide a much more adequate tool for representing real data structures. In this study, we use Fuzzy C-Mean (FCM) algorithm, given by Bezdek (1981). The FCM algorithm minimizes the objective function for the partition of data set, $x=\left[x_{1}\right.$, $\left.x_{2}, \ldots, x_{n}\right]^{\mathrm{T}}$, given by Equation 10 (Sayadi et al., 2007):
$J_{m}(u, v)=\sum_{i=1}^{c} \sum_{k=1}^{n} u_{i, k}^{m}\left\|x_{k}-v_{i}\right\|^{2}$

where, $c$ is the number of the cluster $(1 \leq c \leq n) ; n$ is the number of samples in the vector $X ; \mu_{i k}$ is the element of the partition matrix $U$ of size $(c \times n)$, in which each member indicates the degree of membership of data vector in cluster $i ; v_{i}$ is the cluster center of the $i^{\text {th }}$ cluster and $m$ is the fuzzifier, $m>1$, which controls the fuzziness of the method. The elements of the partition matrix $U$ is constrained to contain elements in the range $[0,1]$ and it should satisfy the following conditions Equation 11:

$\sum_{i=1}^{c} \mu_{i k}=1 ; 1 \leq k \leq n$

and

$0 \leq \sum_{k=1}^{n} \mu_{i k} \leq n ; 1 \leq i \leq c$

In the framework of the segmentation of an image of size $(N \times M)$, the vector $X$ contains all the gray level of the image, scanned line by line, i.e., $n=N M$. The fuzzy cmeans algorithm performs the partition of the vector $X$ into $c$ fuzzy subsets where $\mu_{i k}$ represents the membership value of $x_{k}$ in cluster $i^{t h}$. The FCM clustering technique can be summarized by the following steps (Sayadi et al., 2007):

Step 1: Initialization (Iteration 0):

- Scan the image line by line to construct the vector $X$ containing all the gray level of the image

- Randomly initialize the centers of the classes vector $V^{(0)}$

From the iteration $t=1$ to the end of the algorithm:

Step 2: Calculate the membership matrix $U^{(t)}$ of element $\mu_{i k}$ using Equation 12:

$u_{i k}=\left(\sum_{j=1}^{c}\left(\frac{\left\|x_{k}-v_{i}\right\|}{\left\|x_{k}-v_{j}\right\|}\right)^{\frac{2}{m-1}}\right)^{-1}$

Step 3: Calculate the vector $V^{(t)}=\left[v_{1}, v_{2}, \ldots, v_{c}\right]$ using Equation 13:

$$
v_{i}=\frac{\sum_{k=1}^{n} \mu_{i k}^{m} x_{k}}{\sum_{k=1}^{n} \mu_{i k}^{m}}
$$


Step 4: Convergence test: if $\left\|V^{(t)}-V^{(t-1)}\right\|<\varepsilon$ then stop, otherwise, increase $t$ by one and return to Step 2 . $\varepsilon$ is a chosen positive threshold.

\section{RESULTS}

In this section, we present a few results of the extensive simulations carried out in order to segmentation of the EEG signals image by using fuzzy c-mean. Three cluster centers of the flat EEG given in Table 1. These data are transformed into image form at every time $t$ by using (9). The obtained image forms are segmented into regions by using FCM in Matlab routine program (Fig. 1 and 2 ).

\section{DISCUSSION}

We have shown that the EEG signals during epileptic seizure can be transformed into image form at every time and then it is segmented into regions. The same color on the segmentation at time $t$ show the locations of the source of electric current which has nearly equal current strength.

Table 1. Position and electrical potential of the cluster centers at two different times

\begin{tabular}{|c|c|c|c|}
\hline \multirow[b]{2}{*}{$\begin{array}{l}\text { Time } \\
\text { (second) }\end{array}$} & \multicolumn{2}{|c|}{ Position } & \multirow[b]{2}{*}{$\begin{array}{l}\text { Electrical potential } \\
\qquad(\mu \mathrm{V})\end{array}$} \\
\hline & $\mathrm{X}$ & y & \\
\hline \multirow{8}{*}{$9^{\text {th }}$} & 3.0186 & -4.03605 & 90.1207110 \\
\hline & -7.9024 & -2.56866 & 353.3531700 \\
\hline & -7.9033 & 2.56786 & 650.2743200 \\
\hline & 2.3517 & -0.20946 & 7.2889276 \\
\hline & 4.2557 & 5.63958 & 45.7452240 \\
\hline & -3.6039 & -0.34369 & 113.3302500 \\
\hline & -4.8150 & 6.45112 & 239.9282700 \\
\hline & -2.5861 & -2.57170 & 199.6538100 \\
\hline \multirow{3}{*}{$14^{\text {th }}$} & 4.4190 & -5.56860 & 417.6533000 \\
\hline & -7.4909 & 2.28810 & 61.3587620 \\
\hline & -1.9327 & -3.94050 & 11.7119320 \\
\hline
\end{tabular}

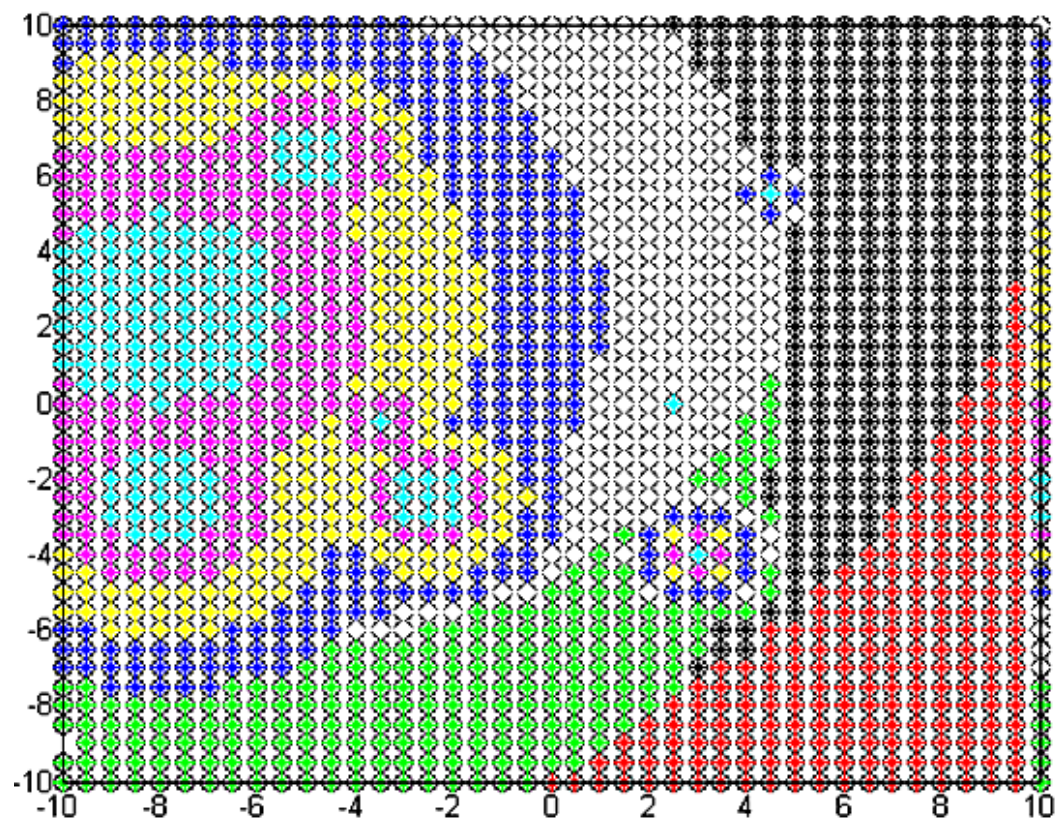

Fig. 1. Segmentation of EEG signals at time 9th 


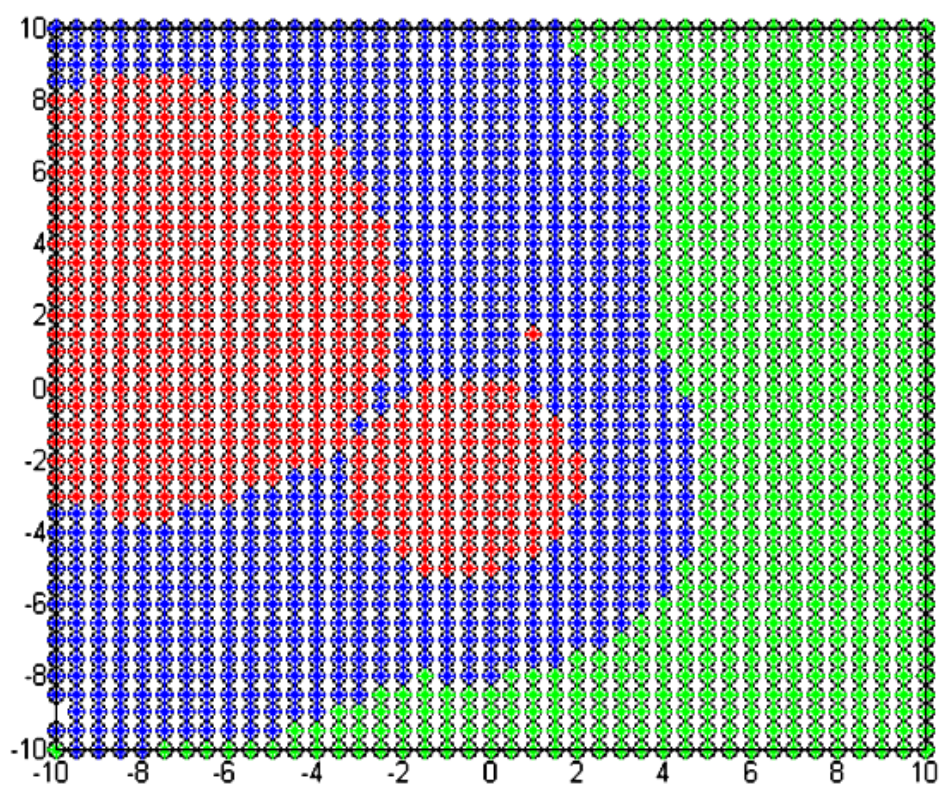

Fig. 2. Segmentation of EEG signals at time 14th

\section{CONCLUSION}

This study will enable us to predict te locations of current sources which have similar strengths. Further research can use other segmentation method to compare the results of this study.

\section{ACKNOWLEDGMENT}

The researchers gratefully acknowledge the reviewers for the constructive comments.

\section{ADDITIONAL INFORMATION}

\subsection{Funding Information}

This research work was funded by the Government of South Sulawesi Province, Indonesia and Universiti Teknologi Malaysia

\subsection{Author's Contributions}

Muhammad Abdy prepared, developed and published of this manuscript, and Tahir Ahmad revised and approved the final version of this manuscript.

\subsection{Ethics}

There are no ethical issues involved because this is our original research work.

\section{REFERENCES}

Abdy, M., 2014. Fuzzy topological digital space and image form of fEEG during epileptic seizures. PhD Thesis, Universiti Teknologi Malaysia, Johor, Malaysia.

Ahmad, T. and T.L. Ken, 2010, Topological conjugacy between seizure and flat electroencephalography. Am. J. Applied Sci., 7: 1470-1476. DOI: 10.3844/ajassp.2010.1470.1476

Adeli, H., 2007. A wavelet-chaos methodology for analysis of EEGs and EEG Sub bands to detect seizure and epilepsy. IEEE Trans. Biomed. Eng., 54: 205-211. DOI: 10.1109/TBME.2006.886855

Bezdek, J.C., 1981. Pattern Recognition with Fuzzy Objective Function Algorithms. 1st Edn., Plenum, New York, pp: 256.

Binjadhnan, F.A.M. and T. Ahmad, 2010, EEG signals during epileptic seizure as a semigroup of upper triangular matrices. Am. J. Applied Sci., 7: 540-544. DOI: 10.3844/ajassp.2010.540.544

Bloch, I., H. Maituri and M. Anvari, 1997. Fuzzy adjacency between image objects. Int. J. Uncertainty Fuzziness Knowledge-Based Syst., 5: 615-653. DOI: $10.1142 / S 0218488597000476$

Demko, C. and E.H. Zahza, 1995. Image understanding using fuzzy isomorphism of fuzzy structures. Proceedings of the IEEE International Conference on Fuzzy System, Mar. 20-24, IEEE Xplore Press, Yokohama, Japan, pp: 1663-1672. DOI: 10.1109/FUZZY.1995.409900 
Engel, J.J.R., P.C. Van Ness, T.B. Rasmussen and L.M. Ojemann, 1993. Outcome with Respect to Epileptic Seizures. In: Surgical Treatment of the Epilepsies. J. Engel Jr., (Ed.), Raven Press, New York, ISBN-10: 0881679887, pp: 609-622.

Guo, L., D. Rivero and A. Pazos, 2010, Epileptic seizure detection using multiwavelet transform based approximate entropy and artificial neural networks. J. Neusci. Meth., 193: 156-163. DOI: 10.1016/j.jneumeth.2010.08.030, PMID: 20817036

Sayadi, M., L. Tlig and F. Fnaiech, 2007. A new texture segmentation method based on the fuzzy c-mean algorithm and statistical features. Applied Math. Sci., 1: 2999-3007.
Subasi, A., 2005. Epileptic seizure detection using dynamic wavelet network. Expert Syst. Applic., 29: 343-355. DOI: 10.1016/j.eswa.2005.04.007

Zakaria, F., 2008. Dynamic profiling of EEG data during seizure using fuzzy information. $\mathrm{PhD}$ Thesis, Universiti Teknologi Malaysia, Johor, Malaysia.

Zhanga, Z., H. Kawabatab and Z. Liu, 2001. Electroencephalogram analysis using fast wavelet transforms. Comput. Biol. Medicine, 31: 429-440. DOI: $10.1016 / \mathrm{S} 0010-4825(01) 00019-1$ 